\title{
Image Analysis for MRI-Based Brain Tumor Classification Using Deep Learning
}

\author{
Krisna Nuresa Qodri ${ }^{1}$, Indah Soesanti ${ }^{2}$, Hanung Adi Nugroho ${ }^{3}$
}

\begin{abstract}
Tumors are cells that grow abnormally and uncontrollably, whereas brain tumors are abnormally growing cells growing in or near the brain. It is estimated that 23,890 adults $(13,590$ males and 10,300 females) in the United States and 3,540 children under the age of 15 would be diagnosed with a brain tumor. Meanwhile, there are over 250 cases in Indonesia of patients afflicted with brain tumors, both adults and infants. The doctor or medical personnel usually conducted a radiological test that commonly performed using magnetic resonance image (MRI) to identify the brain tumor. From several studies, each researcher claims that the results of their proposed method can detect brain tumors with high accuracy; however, there are still flaws in their methods. This paper will discuss the classification of MRI-based brain tumors using deep learning and transfer learning. Transfer learning allows for various domains, functions, and distributions used in training and research. This research used a public dataset. The dataset comprises 253 images, divided into 98 tumor-free brain images and 155 tumor images. Residual Network (ResNet), Neural Architecture Search Network (NASNet), Xception, DenseNet, and Visual Geometry Group (VGG) are the techniques that will use in this paper. The results got to show that the ResNet50 model gets $96 \%$ for the accuracy, and VGG16 gets $96 \%$ for the accuracy. The results obtained indicate that transfer learning can handle medical images.
\end{abstract}

Keyword-Tumor, Brain Tumors, Magnetic Resonance Image (MRI), Accuracy, Deep Learning, Transfer Learning, VGG16, ResNet50.

\section{INTRODUCTION}

The brain is a very important part of the human body. This is because the brain acts as a power system for all members of the human body, such as hand gestures, foot movements, eyeballs, and other vital parts of the body [1]. Brain activity may be impaired if there is interference in the brain, one of which is the involvement of a tumor. Tumors are cells that grow abnormally and uncontrollably, whereas brain tumors are cells that grow abnormally in or near the brain [2]. There are two types of brain tumors, namely benign tumors and malignant tumors.

Benign tumors are a type of tumor that is not too destructive. These tumors arise from cells in or near the brain, do not contain cancer cells, expand slowly, and normally have strong borders, and do not spread to other tissues [2]. In comparison, malignant tumors are malignant brain tumors containing cancer cells and have no clear borders. This type of tumor can be life-

1,2,3 Department of Electrical and Information Engineering, Faculty of Engineering, Universitas Gadjah Mada, Grafika Street 2 Yogyakarta $55281 \quad$ Indonesia (e-mail: ${ }^{1}$ krisna.nuresa.q@mail.ugm.ac.id, $\quad 2$ indahsoesanti@ugm.ac.id; 3adinugroho@ugm.ac.id) threatening since it develops quickly and can penetrate the underlying brain tissue [2].

According to reports, about 23,890 adults (13,590 males and 10,300 females) in the United States and nearly 3,540 children under the age of 15 are diagnosed with brain tumors [3]. Meanwhile, there are over 250 cases of patients afflicted with brain tumors, both adults and infants, in Indonesia [4].

Radiological examination using magnetic resonance imaging (MRI) is needed to determine whether a person has a tumor or not. MRI is selected because it can observe a distinction of soft tissues such as white matter and gray matter [4]. The MRI analysis will create a picture that represents the brain. Several factors that influence the diagnosis of brain tumors include the skill of the medical team, brain anatomy, size, and form of the tumor. These factors increase the difficulty in identifying brain tumors [5].

Technological advances, especially in artificial intelligence and machine learning, have a significant influence on the medical field; one of them is the recognition of medical images. The Convolution Neural Network (CNN) is one technique used to process or detect brain tumors using MRI images [6]. CNN is a model of deep learning to process data that has a grid structure such as images inspired by the organization of the visual cortex of animals [7], [8] and is designed to extract details locally on an image and has the potential to store information spatially. The efficiency produced by this approach is excellent since this method has a reasonably good biased capability [9]. The development of this approach has inspired different types of architecture, including Residual Network (ResNet), Network in Network (NiN), VGG, and GoogleNet (Inception module). Transfer learning is the knowledge that is extracted from different sources and applied to other fields [10]. Transfer learning allows for various domains, functions, and distributions to be used in training and research [10]. Research [11], used ImageNet pre-trained ALexNet to PASCAL dataset [12], the results of the detection and segmentation performance improved significantly. Therefore, this paper will discuss the classification of brain tumors using deep learning and transfer learning.

\section{DATASET AND RELATED WORK}

There are several studies on brain tumor detection using brain MRI that were performed before. The Discrete Wavelet Transform (DWT), Continuous Wavelet Transform (CWT), and Support Vector Machine (SVM) methods are used to detect brain tumors [13]. This method gets high enough results to detect brain tumors; however, there are still weaknesses in computation. CNN and SVM methods are used to identify brain tumors [14]. The method obtains quite high result for the SVM method. Even though the results are quite high, the 
configuration of the created model is not included, and the dataset is not explained, so the results obtained are biased. Also, the proposed fractional calculus is used to detect benign brain tumors [15]. The result shows that the method produces low computation. Although the method produces low computation, the accuracy that is obtained using these methods is not explained. A study explains that the Watershed Dynamic Angle Projection - Convolution Neural Network (WDAPP-CNN) method is used for the segmentation and classification of brain tumors [16]. The proposed algorithm gets high enough results for sensitivity and efficiency. However, it only focuses on the sensitivity and efficiency and not on the accuracy, so the accuracy is unknown. A study proposes the Faster R-CNN method for the detection of brain tumors using brain MRI images [17]. This method also produces high accuracy. Even though they get high results, they do not clarify the computation they get. In other studies, Naive Bayes classifier is proposed to detect brain tumors using MRI images [18]. This study claims that these methods $60 \%$ better than the other methods. Although it claims that the method is $60 \%$ better than the other methods, the accuracy obtained is not more than $84 \%$. An Artificial Neural Network (ANN) is proposed to detect brain tumors [19]. This method claims to get high enough results to detect brain tumors. Despite getting high results, the proposed method cannot directly detect tumors, the user must select each stage from pre-processing to tumor segmentation, and then classification can be carried out. Furthermore, the computations to detect tumors are not explained. A "multiple threshold object counting" technique is proposed for detecting brain tumors [20]. The technique claims to be able to detect brain tumors with good accuracy. Even though the results are quite high, this method only able to detect the position of the tumor and cannot recognize whether the image contains the tumor or not. The SVM algorithm is proposed for detecting brain tumors [21]. The algorithm claims to be able to detect tumors with high accuracy. Even though the results are quite high, this method only able to detect the position of the tumor and cannot recognize the image that contained the tumor or not. The combination of the feature extraction algorithm and CNNSoftMax is proposed for detecting brain tumors [22]. This method claims to be able to obtain high accuracy results. Even though it gets high results, it does not clarify the computation obtained.

Deep learning has been used in many fields, such as omics, bioimaging, medical imaging, brain-machine interfaces, or body machine interfaces [9]. The most famous Deep Learning architecture for image analysis is CNN [9]. A study was conducted for malignancy determination of lung nodules using 3D CNN architecture [23]. CNN is proposed to segmentation and multi-structure cardiac diagnosis [24]. CNN is also proposed to detect early mild cognitive impairment (MCI) [25]. CNN is proposed for nuclei detection [26]. Some studies above use deep learning to solve their respective problems. The deep learning architecture that they use is CNN. The method can give quite excellent results.

From the research mentioned above, many studies are conducted to detect brain tumors, but there are still some
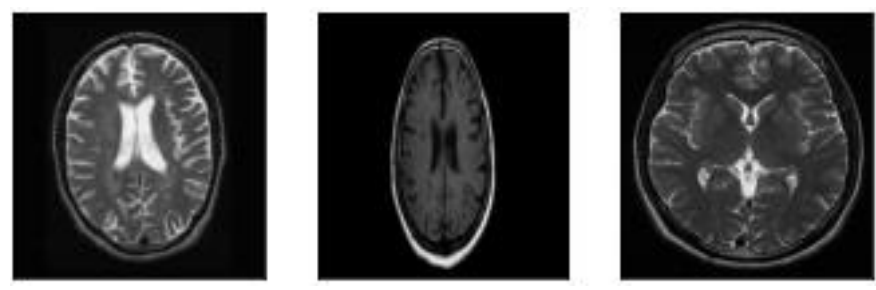

Fig. 1 Normal brain MRI image.
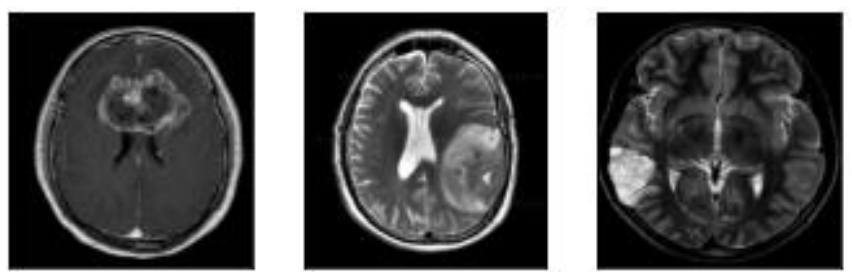

Fig. 2 Abnormal brain MRI image.

shortcomings of these studies. Therefore, in this study, the researchers will discuss the detection of brain tumors. The model that the researchers will use in this research is a model based on the $\mathrm{CNN}$ architecture, and the researchers use transfer learning to classify tumors using MRI brain images. Some models that the researchers will use in this research include Residual Network (ResNet), Neural Architecture Search Network (NASNet), Xception, DenseNet, and Visual Geometry Group (VGG).

The researchers used a public dataset for this research [27]. The dataset contained MRI images of the brain, either normal images (no tumor) or abnormal images (there are tumors). The dataset had a .jpg extension for each image. The dataset comprised 253 images and was divided into 98 tumor-free brain images and 155 tumor images. An example of a normal brain MRI image (no tumor) can be seen in Fig. 1, whereas a nonnormal brain MRI image (there is a tumor) can be seen in Fig. 2.

\section{RESEARCH METHOD}

In this research, the researchers analyze and evaluate the impact of different $\mathrm{CNN}$ architectures.

\section{A. Convolutional Neural Network Architectures (CNN)}

The Convolutional Neural Network (CNN) is a form of deep learning that is commonly used [28]. It is a deep learning algorithm built based on the Multilayer Perceptron (MLP) for processing data as two-dimensional imagery. There are several models based on the $\mathrm{CNN}$ architecture, that is:

1) Residual Network (ResNet): ResNet was introduced in 2016 [29]. ResNet consists of several piled "Residual Units" [29]. Each unit (Fig. 3) can be represented in the following general form:

$$
\begin{gathered}
y_{l}=h\left(x_{l}\right)+\mathcal{F}\left(x_{l}, \mathcal{W}_{l}\right) \\
x_{l}+1=f\left(y_{l}\right)
\end{gathered}
$$

where the input is $x_{l}$ and the output is $x_{l}+1$ of the $l$-th unit, and the residual function is $\mathcal{F}$. The identity mapping is $h\left(x_{l}\right)=$ $x_{l}$ [29] and the ReLu function is $f$ [30]. ResNets over 100-layer 


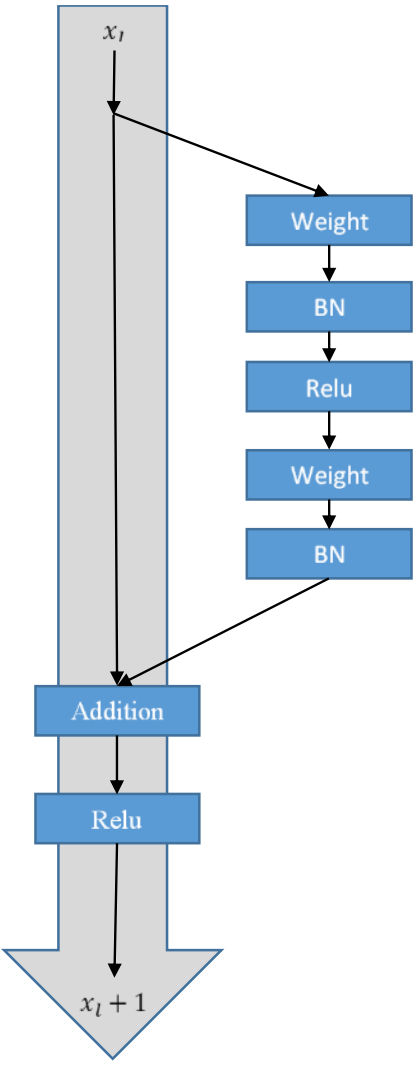

Fig. 3 Residual unit.

depth results showed state-of-the-art accuracy for many demanding image classification at ImageNet [31] and MS COCO [32] competitions. The main characteristic of ResNets is to learn the additive residual function $\mathcal{F}$ with respect to $h\left(x_{l}\right)$, with the main option of using an identity mapping $h\left(x_{l}\right)=x_{l}$. This was achieved by adding an identification skip link ("shortcut").

2) Xception: 36 convolutional layers in the Xception architecture form the network's function extraction foundation. Xception itself was introduced in 2017 [33]. Xception is a linear, residual-connected, depth-separable stack of convolution layers. In the ImageNet dataset [34] after InceptionresnetV2 [35] and NasNet Large [36], Xception got the third-best performance. This made it easier to change the model.

3) Visual Geometry Group (VGG): The VGG architecture was introduced in 2015 [37]. The accuracy results obtained from a data set of more than 14 million images included in 1000 classes on ImageNet, the model was able to get an accuracy of $92.7 \%$. The input from layer 1 had a size of 224 x 224 RGB images. VGG used a filter that was $3 \times 3$ in size relative to a larger filter. VGG used Multi-Scale for training and testing. Apart from using the Multi-Scale test, VGG also used dense testing. The error rate could be lowered by $6.8 \%$.

4) Neural Architecture Search Network (NASNet): The NASNet architecture comprises Controller Recurrent Neural Network (CRNN) and CNN [38]. In their research [36], the NASNet algorithm operates by choosing the best cells using the
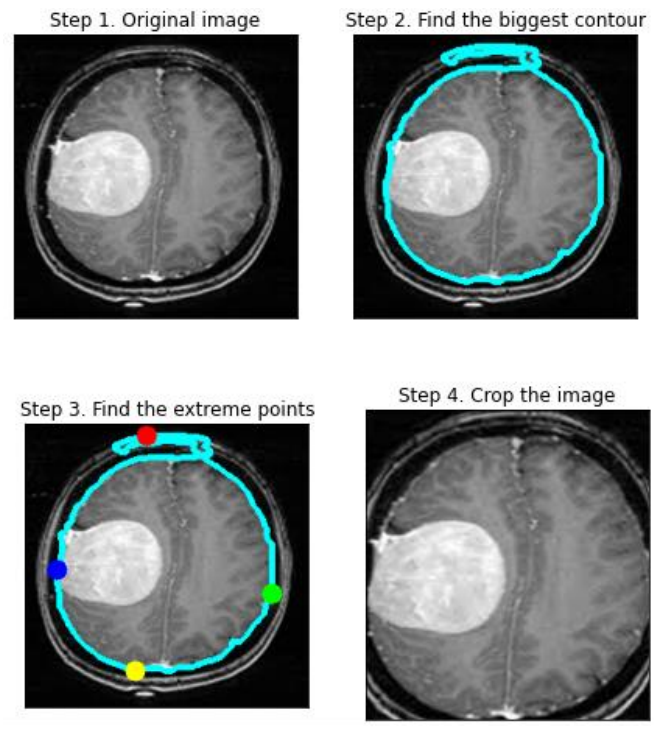

Fig. 4 Image cropping process.
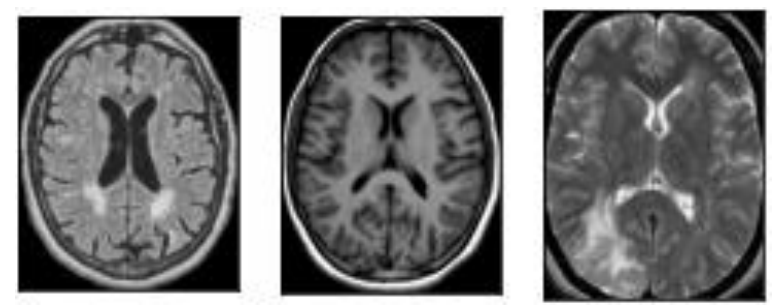

Fig. 5 Normal brain image after the cropping process.
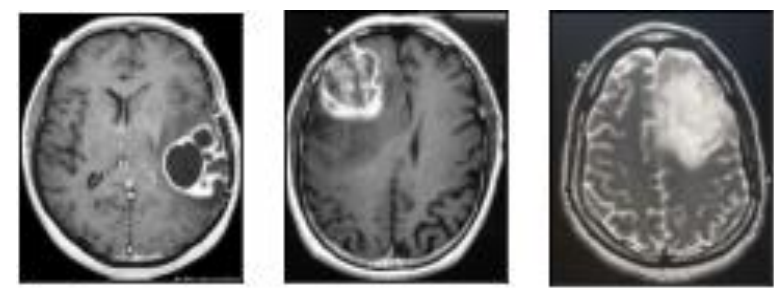

Fig. 6 Abnormal brain images after the cropping process.

reinforcement learning method. NASNet architecture used two types of image input sizes, namely 331 x 331 and 224 x 224 sizes.

DenseNet: Several studies have shown that CNNs can increase performance if the layers close to the input and near the output have shorter connections. [39]. As a result, a new model called DenseNet was developed, which links each layer feed-forward to each other. Inputs were used for all the previous layers of the feature maps, input to each layer of the DenseNet feature maps, and their own feature maps [40].

\section{B. Pre-trained CNN}

The researchers adopted six deep CNN architectures, which were ResNet50, Xception, DenseNet, VGG19, VGG16, and NasNetLarge as the feature extractors of the proposed method for tumor detection based on brain MRI. The model was trained using a nature image (ImageNet) and could be extended to the computational transition of learning to remove discriminatory features from biomedical images [41]. 


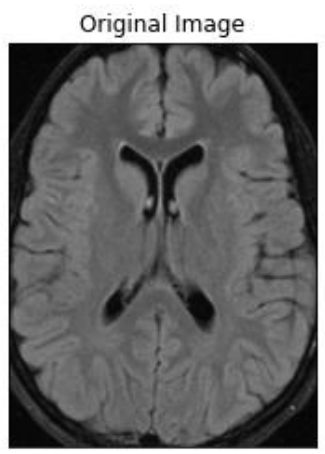

Augemented Images
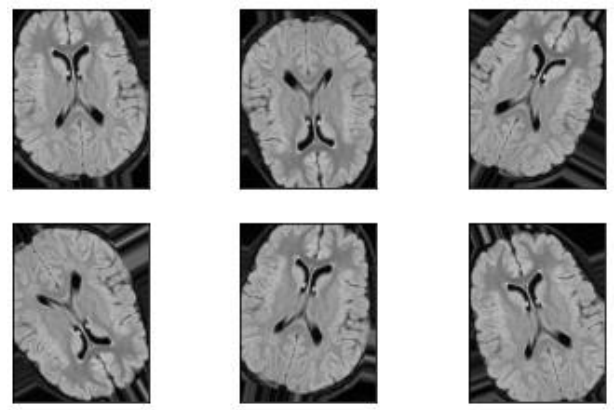

Fig. 7 The results of the augmented image.

\section{Transfer Learning}

Transfer learning is the knowledge that is extracted from different sources and applies to other fields [10]. Transfer learning allows for various domains, functions, and distributions used in training and research [10]. In their research [11], using ImageNet pre-trained ALexNet to PASCAL dataset [12], the results of the detection and segmentation performance improves significantly. Similar results are obtained [42], [43]. In their study, they use transfer learning and are able to enhance the efficiency of their proposed model. According to the few studies that obtain outstanding performance after using transfer learning, the researchers believe that considering the variations in images, CNNs that are completely trained on ImageNet can still be transferred to make recognition using medical images.

\section{Loss Function}

One of the essential components in the design of a neural network is the estimation of the output of the entire system or the measurement of errors in the system. The type of loss function used depends on the type of task of the model being done. In the case of neural networks with regression or classification tasks, the loss function is defined as $l$ for one training data and $\mathrm{L}$ for dataset $x$.

$$
L(x, W)=\frac{1}{N} \sum_{i=0}^{N} l\left(x_{i}, W\right) .
$$

Minimization of loss functions for model parameters is a problem of optimization, as described in the following equation.

$$
W=\underset{W}{\arg \min } L(x, W)
$$

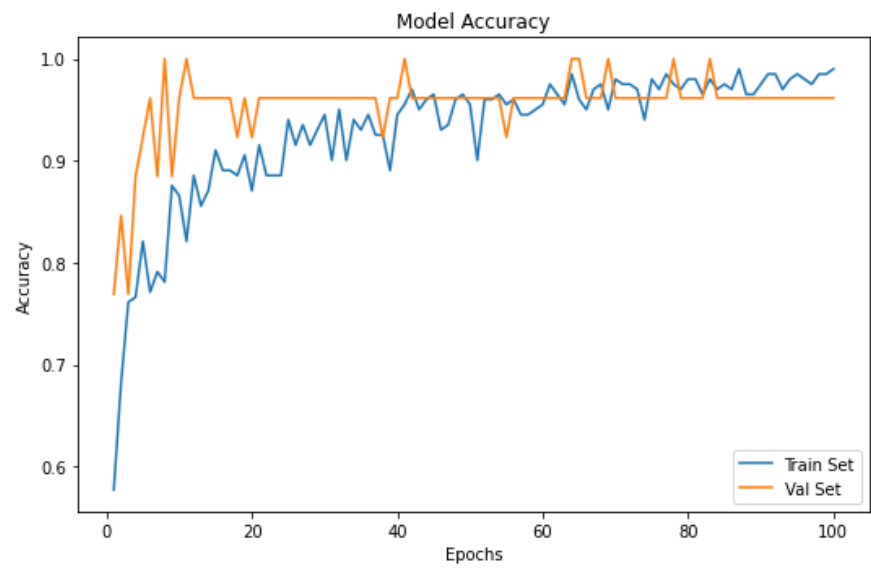

Fig. 8 Results of training and validation of the ResNet50 model.

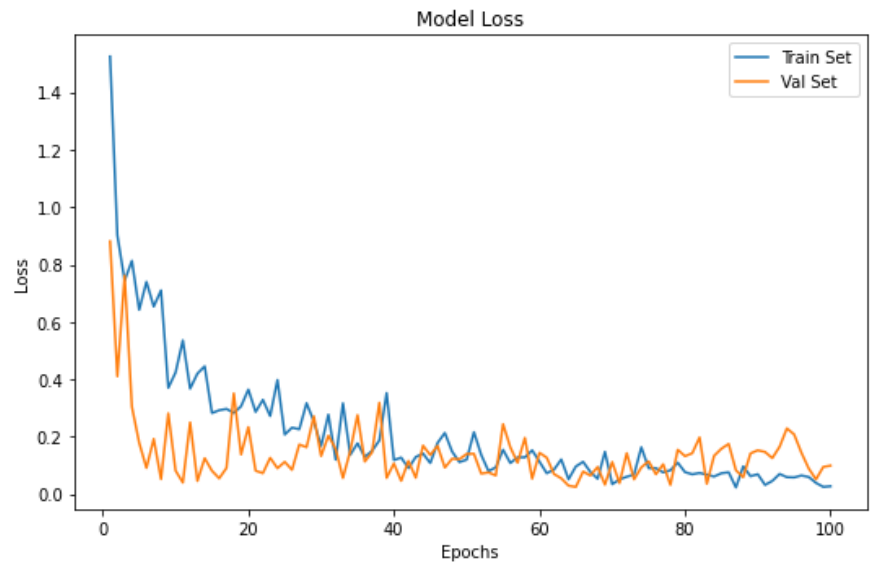

Fig. 9 ResNet50 model loss data graph.

\section{RESUlTS AND DisCUSSION}

In this section, the researchers will compare and evaluate the performance of six CNN models (ResNet50, Xception, VGG19, VGG16, NASNetLarge, and DenseNet) for detecting brain tumors using brain MRI using a dataset.

In the early stages, researchers pre-processed the MRI images of the brain. At this stage, researchers normalized the data by cropping the image and resizing the image. Fig. 4 shows the results of before and after cropping and resizing images. Fig. 5 and Fig. 6 show the images after cropping and resizing process.

Fig. 4 explains how to crop the image. The first step (Step 1) is to prepare the image. Then the second step (Step 2) is to find the biggest contour. The third step (Step 3) is to find the extreme points based on the biggest contour. Finally, the last step (Step 4) is to crop the image that corresponds to the extreme points. Next, researchers performed data augmentation. The purpose of data augmentation was to increase the amount of data from the "training" data. researchers used a "random transformation," and used 30 for "rotation rang.". Fig. 7 shows the results of the augmented data.

Next, researchers split the dataset with the configuration: $80 \%$ for training, $10 \%$ for validation, and $10 \%$ for testing. Then researchers built the model for each architecture (ResNet50, VGG19, VGG16, NASNetLarge, Xception, and DenseNet192) 


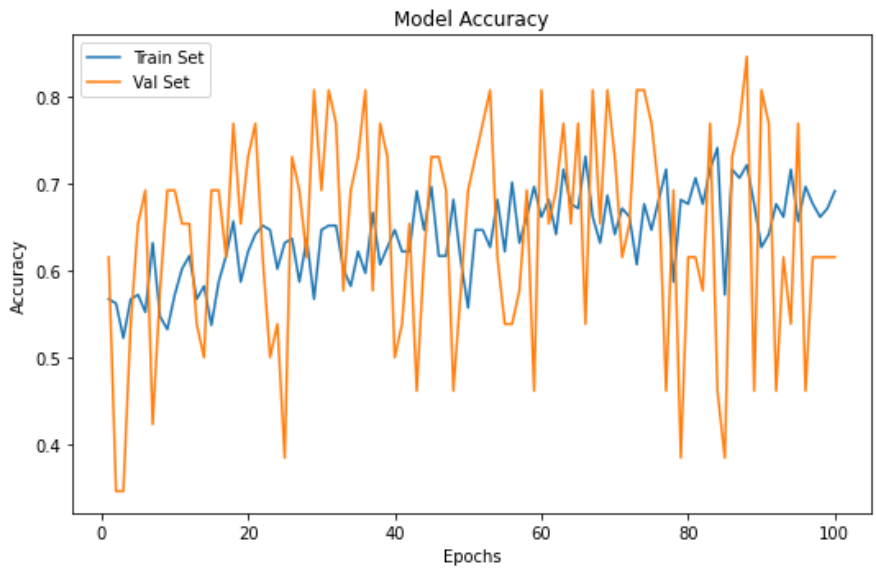

Fig. 10 Results of training and validation of the NASNetLarge model.

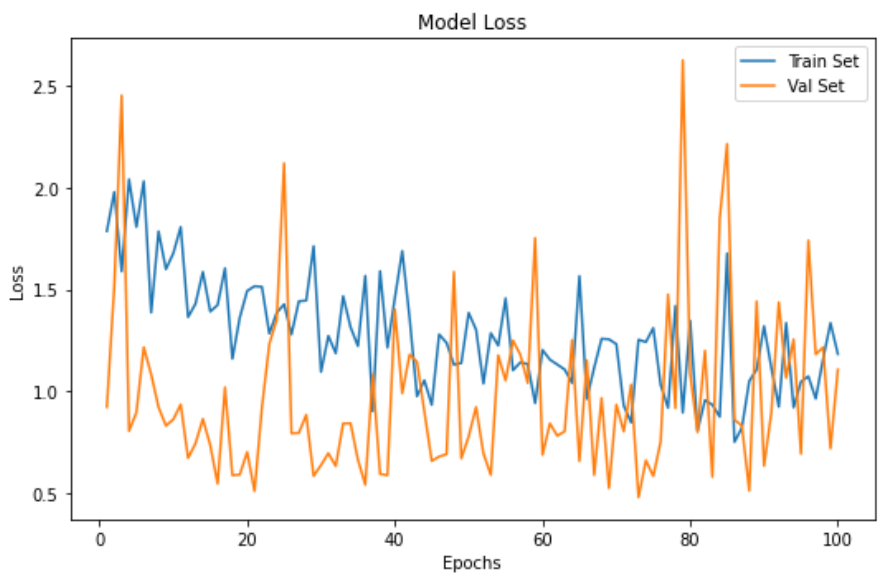

Fig. 11 NASNetLarge model loss data graph.

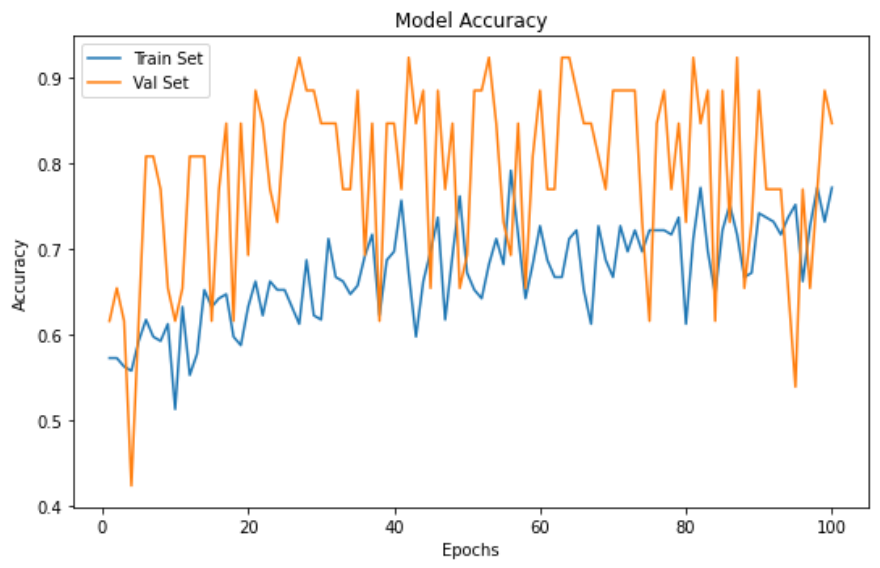

Fig. 12 Results of training and validation of the DenseNet192 model.

with the configuration: researchers used 32 for the training batch size, 16 for the validation batch size, and 100 for the epoch, "max" for the pooling, "imagenet" for the weights and for the optimizer researchers used "RMSprop". Fig. 8 to Fig. 15 shows the results of each model. Fig. 8 and Fig. 9 are the graphs by the ResNet50 model.

The ResNet model obtained high results in both training and validation. The training accuracy got $99 \%$ and $96 \%$ for validation accuracy. Data loss using the ResNet50 model (Fig. 9) shows that the model has low data loss. Then with the

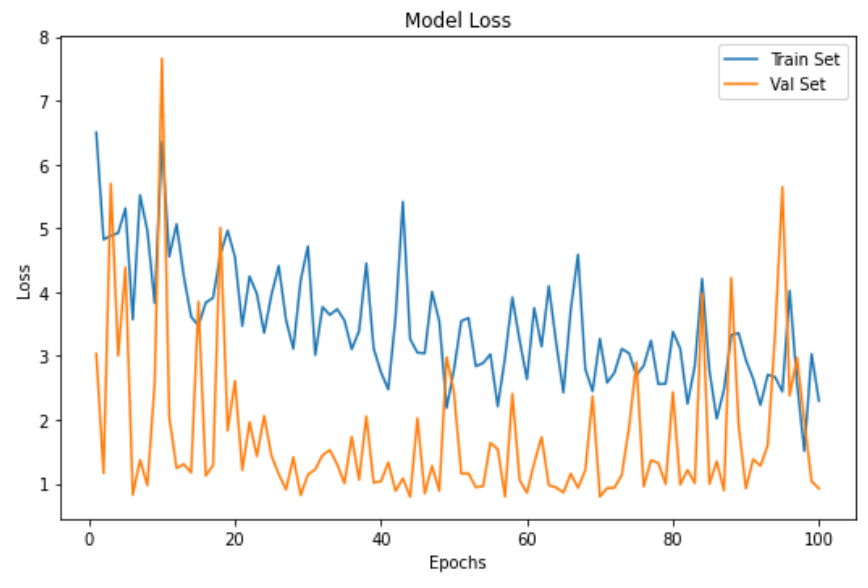

Fig. 13 DenseNet192 model loss data graph.

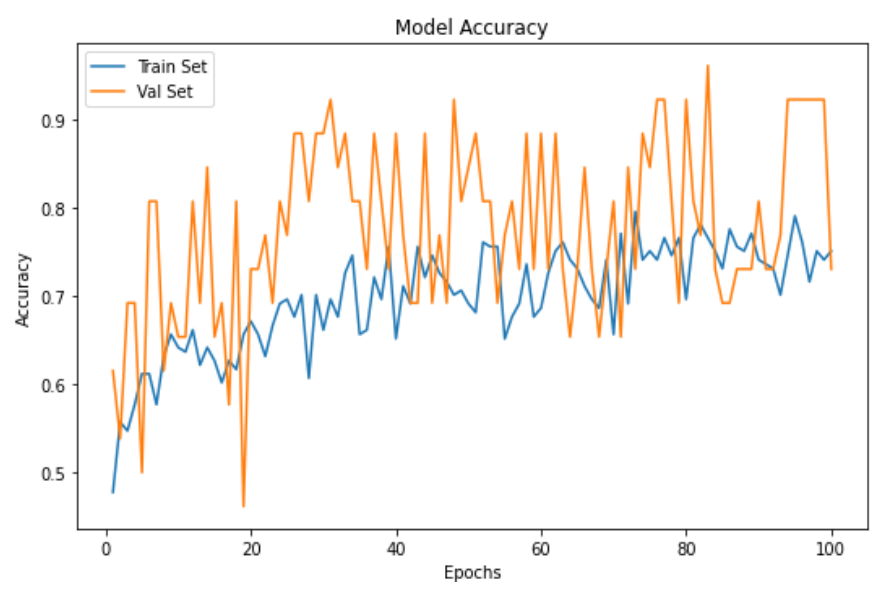

Fig. 14 Results of training and validation of the Xception model.

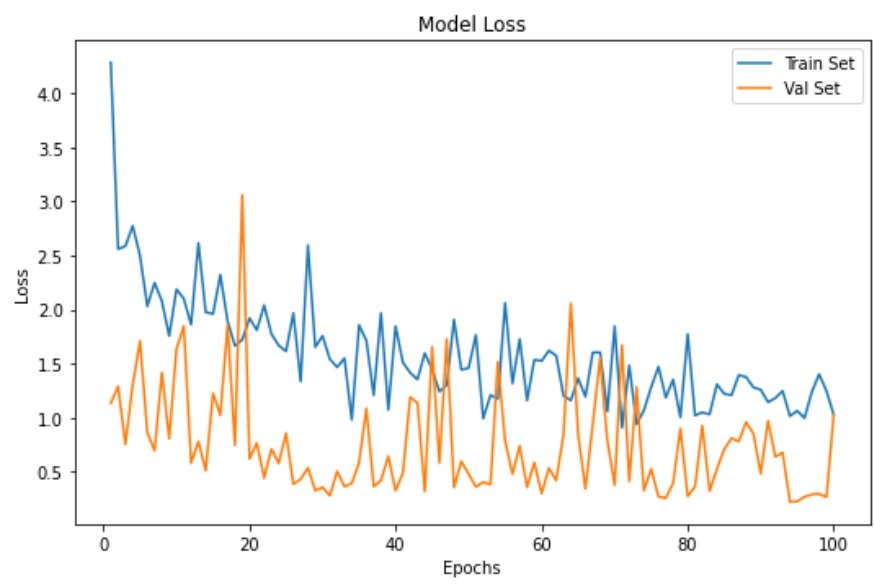

Fig. 15 Xception model loss data graph.

NASNetLarge model, the results can be seen in Fig. 10 and Fig. 11.

The NASNetLarge model obtained poor results on both training and validation. The training accuracy obtained by this model was $62 \%$ and got $62 \%$ accuracy for the validation, while data loss can be seen in Fig. 11. Data loss using the NASNetLarge model shows that the model has a high enough data loss. The results obtained using the DenseNet192 model can be seen in Fig. 12 and Fig. 13. 


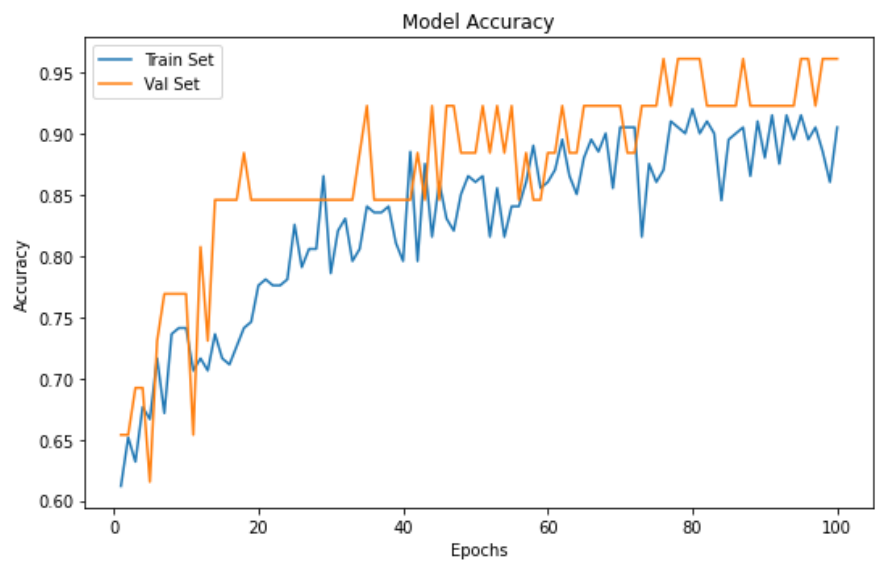

Fig. 16 Results of training and validation of the VGG16 model.

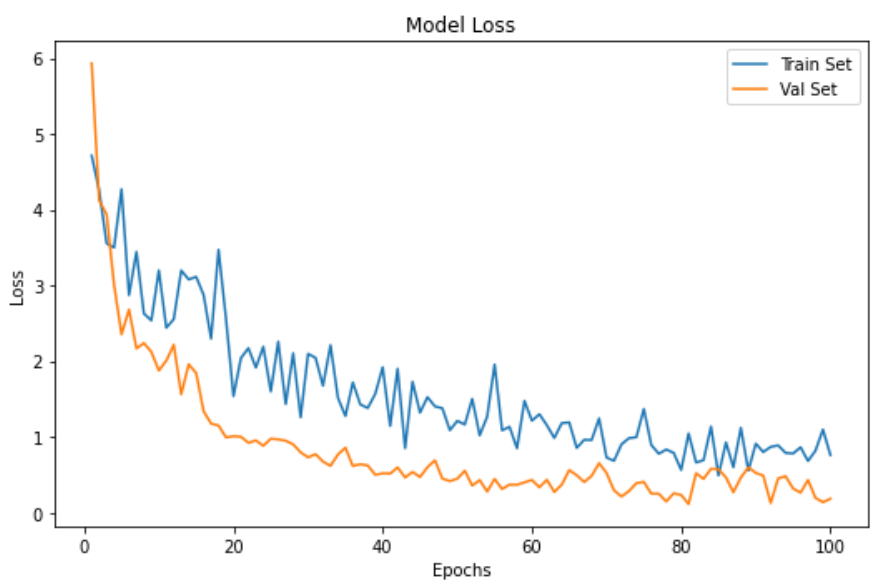

Fig. 17 VGG16 model loss data graph.

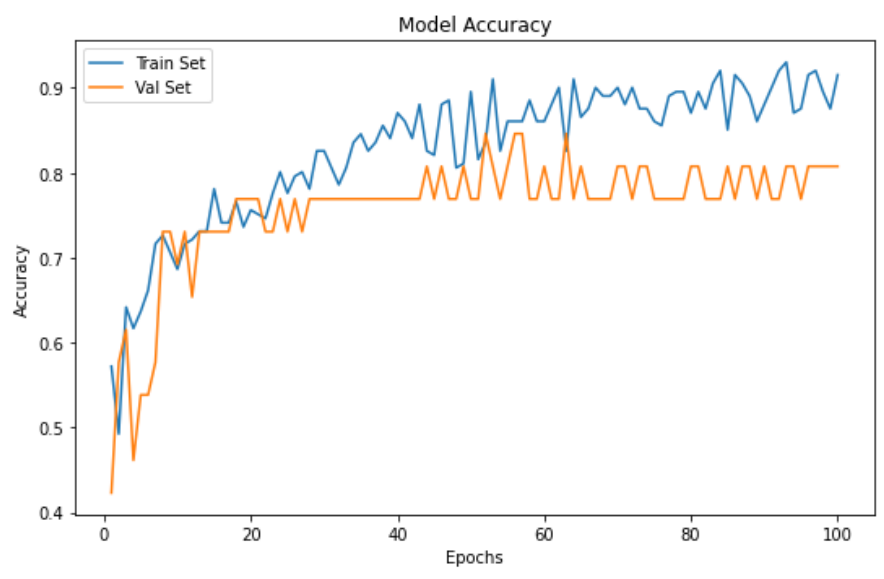

Fig. 18 Results of training and validation of the VGG19 model.

The DenseNet192 model got fairly low results in both training and validation. The training accuracy obtained by this model was $78 \%$ and got $88 \%$ accuracy for validation, while data loss can be seen in Fig. 13. Data loss using the NASNetLarge model shows that the model has a high enough data loss. The results obtained using the Xception model can be seen in Fig. 14 and Fig. 15.

The Xception model also got low results in both training and validation. The training accuracy obtained by this model was $74 \%$ and got $73 \%$ accuracy for validation, while the data loss

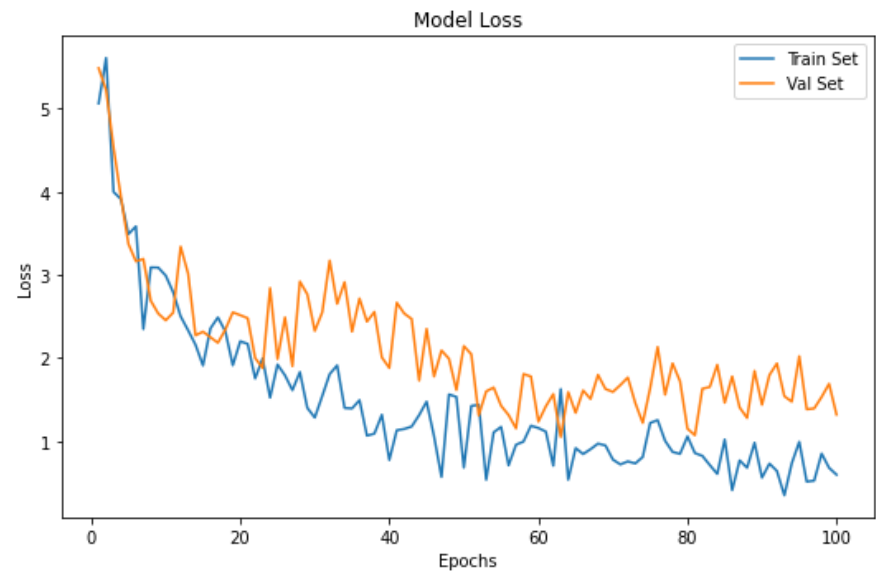

Fig. 19 VGG19 model loss data graph.

TABLE I

ACCURACY RESULTS FOR EACH MODEL

\begin{tabular}{|l|l|c|c|c|}
\hline No & Model & $\begin{array}{c}\text { Training } \\
\text { Accuracy }\end{array}$ & $\begin{array}{c}\text { Validation } \\
\text { Accuracy }\end{array}$ & $\begin{array}{c}\text { Test } \\
\text { Accuracy }\end{array}$ \\
\hline 1 & ResNet50 & $\mathbf{9 9 \%}$ & $\mathbf{9 6 \%}$ & $\mathbf{8 5 \%}$ \\
\hline 2 & NASNetLarge & $62 \%$ & $62 \%$ & $69 \%$ \\
\hline 3 & Xception & $74 \%$ & $73 \%$ & $69 \%$ \\
\hline 4 & DenseNet192 & $77 \%$ & $88 \%$ & $81 \%$ \\
\hline 5 & VGG16 & $97 \%$ & $\mathbf{9 6 \%}$ & $\mathbf{8 5 \%}$ \\
\hline 6 & VGG19 & $97 \%$ & $85 \%$ & $81 \%$ \\
\hline
\end{tabular}

TABLE II

SENSITIVITY PRECISION AND SPECIFICITY FOR EACH MODEL

\begin{tabular}{|l|l|c|c|c|}
\hline No & \multicolumn{1}{|c|}{ Model } & Sensitivity & Precision & Specificity \\
\hline 1 & ResNet50 & $\mathbf{0 . 9 4}$ & 0.83 & $\mathbf{0 . 7 0}$ \\
\hline 2 & NASNETLarge & 0.75 & 0.75 & 0.60 \\
\hline 3 & Xception & $\mathbf{0 . 9 4}$ & $\mathbf{0 . 9 0}$ & $\mathbf{0 . 7 0}$ \\
\hline 4 & DenseNet192 & $\mathbf{0 . 9 4}$ & 0.79 & 0.60 \\
\hline 5 & VGG16 & $\mathbf{0 . 9 4}$ & 0.75 & 0.50 \\
\hline 6 & VGG19 & $\mathbf{0 . 9 4}$ & 0.79 & 0.60 \\
\hline
\end{tabular}

TABLE III

COMPUTATIONAL TIME FOR EACH MODEL

\begin{tabular}{|l|l|c|}
\hline No & \multicolumn{1}{|c|}{ Model } & $\begin{array}{c}\text { Computational } \\
\text { Time }\end{array}$ \\
\hline 1 & ResNet50 & $310 \mathrm{~s}$ \\
\hline 2 & NASNETLarge & $419 \mathrm{~s}$ \\
\hline 3 & Xception & $304 \mathrm{~s}$ \\
\hline 4 & DenseNet192 & $311 \mathrm{~s}$ \\
\hline 5 & VGG16 & $\mathbf{3 0 2} \mathbf{s}$ \\
\hline 6 & VGG19 & $\mathbf{3 0 2} \mathbf{s}$ \\
\hline
\end{tabular}

can be seen in Fig. 15. Data loss using the Xception model shows that the model has a high enough data loss. Then the VGG16 and VGG19 models can be seen in Fig. 16 to Fig. 19.

Fig. 16 is a graphic display obtained in training and validation of brain tumor detection using MRI using the VGG16 model. While Fig. 17 is a graph display of data loss for the VGG16 model. The results obtained by the VGG16 model got $97 \%$ for the training and $97 \%$ for the validation. Data loss for the model shows that the model has less data loss. Furthermore, the results obtained by the VGG19 model can be seen in Fig. 18 and Fig. 19. 
Fig. 18 is a graph obtained by the VGG19 model during both training and validation. The chart shows that the model gets $97 \%$ for training accuracy and $85 \%$ for validation. In Fig. 19, the data loss graph for the VGG19 model shows that the model still has low data loss.

The results for each model can be seen in Table I to Table III. Table I shows the accuracy results of each model. ResNet50 gets the highest accuracy for training, but the validation and testing accuracy ResNet50 and VGG16 models get the highest accuracy. Table II describes the sensitivity, specificity, and precision for each model. For sensitivity, almost all models get the same result, which is 0.94 , except for the NASNetLarge model. Then, the Xception model gets the highest for precision. The models that get the highest results for the specificity are ResNet50 and Exception, which is each model, gets 0.70 . The computational time for each model for 100 iterations can be seen in Table III. The table shows that the VGG16 and VGG19 models produce the lowest computational time, which is $302-$ second for 100 iterations (3.02-second for each iteration).

\section{CONCLUSION}

Several techniques have been used to identify brain tumors. Any of these studies have shown that transfer learning can be used for recognition using medical data such as MRI images. The results obtained by each model show that the VGG16 and ResNet50 models are capable of achieving high enough results where the accuracy of the results obtained by VGG16 during the validation test is $96 \%$, and the validation test of the ResNet50 model is capable of obtaining an accuracy of $96 \%$. Although ResNet50 and VGG16 obtain high accuracy results, the Xception model gets high results for sensitivity, precision, and specification. These results show that transfer learning can be used for medical data recognition.

Our future work is to increase the number of images and the number of labels. This is required to assess if the model is still capable of producing successful outcomes with other medical images.

\section{ACKNOWLEDGMENT}

The authors gratefully acknowledge the support of The Ministry of Research and Technology and The Ministry of Education and Culture, Republic of Indonesia.

\section{REFERENCES}

[1] C.F. Hotama P., H.A. Nugroho, and I. Soesanti, "Analisis Citra Otak pada Color-Task dan Word-Task dalam Stroop Task menggunakan Elektroencephanology (EEG)," Thesis, Universitas Gadjah Mada, Yogyakarta, Indonesia, 2014

[2] Cancer Support Community, "Understanding Brain Tumors," in Frankly Speaking About Cancer: Brain Tumors, 2019, p. 7.

[3] (2020) "Brain Tumor: Statistics," [Online], https://www.cancer.net/cancer-types/brain-tumor/statistics\#: :text=This year $\% 2 \mathrm{C}$ an estimated $23 \% 2 \mathrm{C} 890$, lifetime is less than $1 \% 25$, access date: 21-Dec-2020.

[4] A.S. Febrianti, T.A. Sardjono, and A.F. Babgei, "Klasifikasi Tumor Otak pada Citra Magnetic Resonance Image dengan Menggunakan Metode Support Vector Machine," J. Tek. ITS, Vol. 9, No. 1, pp. A118-A123, 2020.

[5] P. Afshar, K.N. Plataniotis, and A. Mohammadi, "Capsule Networks for Brain Tumor Classification Based on MRI Images and Coarse Tumor
Boundaries," ICASSP 2019 - 2019 IEEE Int. Conf. on Acoustics, Speech and Signal Proc. (ICASSP), 2019, pp. 1368-1372.

[6] N. Kumari and L. Gray, "Review of Brain Tumor Segmentation and Classification," 2018 Int. Conf. Curr. Trends Towar. Converging Technol., 2018, pp. 1-6.

[7] K. Fukushima, "Neocognitron: A Self-organizing Neural Network Model for a Mechanism of Pattern Recognition Unaffected by Shift in Position," Biol. Cybern., Vol. 36, pp. 193-202, 1980.

[8] D.H. Hubel and T.N. Wiesel, "Receptive Fields and Functional Architecture of Monkey Striate Cortex," J. Physiol., Vol. 195, No. 1, pp. 215-243, 1968

[9] M. Mahmud, M.S. Kaiser, A. Hussain, and S. Vassanelli, "Applications of Deep Learning and Reinforcement Learning to Biological Data," IEEE Trans. Neural Networks Learn. Syst., Vol. 29, No. 6, pp. 2063-2079, 2018.

[10] S.J. Pan and Q. Yang, "A Survey on Transfer Learning," IEEE Trans. Knowl. Data Eng., Vol. 22, No. 10, pp. 1345-1359, 2010.

[11] R. Girshick, J. Donahue, S. Member, and T. Darrell, "Region-based Convolutional Networks for Accurate Object Detection and Segmentation," IEEE Trans. Pattern Anal. Mach. Intell., Vol. 38, No. 1, pp. $142-158,2015$.

[12] M. Everingham, S.M.A. Eslami, L. Van Gool, C.K.I. Williams, J. Winn, and A. Zisserman, "The PASCAL Visual Object Classes Challenge: A Retrospective," Int. J. Comput. Vis., Vol. 111, No. 1, pp. 98-136, 2015.

[13] M. Gurbină, M. Lascu, and D. Lascu, "Tumor Detection and Classification of MRI Brain Image Using Different Wavelet Transforms and Support Vector Machines," 42nd International Conference on Telecommunications and Signal Processing (TSP), 2019, pp. 505-508.

[14] R. Vinoth and C. Venkatesh, "Segmentation and Detection of Tumor in MRI images Using CNN and SVM Classification," 2018 Conference on Emerging Devices and Smart Systems (ICEDSS), 2018, pp. 21-25.

[15] S.K. Chandra, "Effective Algorithm For Benign Brain Tumor Detection Using Fractional Calculus,” TENCON 2018 - 2018 IEEE Region 10 Conference, 2018, pp. 2408-2413.

[16] T.A. Jemimma and Y.J. Vetharaj, "Watershed Algorithm based DAPP Features for Brain Tumor Segmentation and Classification," 2018 International Conference on Smart Systems and Inventive Technology (ICSSIT), 2018, pp. 155-158.

[17] R. Ezhilarasi and P. Varalakshmi, "Tumor Detection in the Brain Using Faster R-CNN," 2018 2nd International Conference on I-SMAC (IoT in Social, Mobile, Analytics and Cloud), 2018, pp. 388-392.

[18] D. Divyamary, "Brain Tumor Detection from MRI Images Using Naive Classifier," 6th International Conference on Advanced Computing and Communication Systems (ICACCS), 2020, pp. 620-622.

[19] H.E.M. Abdalla and M.Y. Esmail, "Brain Tumor Detection by Using Artificial Neural Network," 2018 International Conference on Computer. Control, Electrical, and Electronics Engineering (ICCCEEE), 2018, pp. $1-6$.

[20] M. Nasor and W. Obaid, "MRI Tumor Detection and Localization by Multiple Threshold Object Counting Technique," 2018 International Conference on Computer and Applications (ICCA), 2018, pp. 158-161.

[21] M.S. Majib and T.M.S. Sazzad, "A Framework to Detect Brain Tumor Cells Using MRI Images," International Congress on Human-Computer Interaction, Optimization and Robotic Applications (HORA), 2020, pp. $1-5$.

[22] M. Siar and M. Teshnehlab, "Brain Tumor Detection Using Deep Neural Network and Machine Learning Algorithm," 9th International Conference on Computer and Knowledge Engineering (ICCKE), 2019, pp. 363-368.

[23] S. Hussein, P. Kandel, C.W. Bolan, M.B. Wallace, and U. Bagci, "Lung and Pancreatic Tumor Characterization in the Deep Learning Era: Novel Supervised and Unsupervised Learning Approaches," IEEE Trans. Med. Imaging, Vol. 38, No. 8, pp. 1777-1787, 2019.

[24] O. Bernard, A. Lalande, C. Zotti, et al., "Deep Learning Techniques for Automatic MRI Cardiac Multi-structures Segmentation and Diagnosis: Is the Problem Solved ?" IEEE Trans. Med. Imaging, Vol. 37, No. 11, pp. 2514-2525, 2018.

[25] T.-E. Kam, H. Zhang, Z. Jiao, and D. Shen, "Deep Learning of Static and 
Dynamic Brain Functional Networks for Early MCI Detection," IEEE Trans. Med. Imaging, Vol. 32, No. 2, pp. 478-487, 2020.

[26] M. Tofighi, T. Guo, J.K.P. Vanamala, and V. Monga, "Prior Information Guided Regularized Deep Learning for Cell Nucleus Detection," IEEE Trans. Med. Imaging, Vol. 38, No. 9, pp. 2047-2058, 2019.

[27] J. Djhonson (2020) "Brain MRI Images for Brain Tumor Detection," [Online], https://www.kaggle.com/jjprotube/brain-mri-images-for-braintumor-detection, access date: 13-Dec-2020.

[28] S. Aggarwal and N. Chugh, "Signal Processing Techniques for Motor Imagery Brain Computer Interface: A Review," Array, Vol. 1-2, pp. 1$12,2019$.

[29] K. He, X. Zhang, S. Ren, and J. Sun, "Deep Residual Learning for Image Recognition," IEEE Conference on Computer Vision and Pattern Recognition (CVPR), 2016, pp. 770-778.

[30] V. Nair and G.E. Hinton, "Rectified Linear Units Improve Restricted Boltzmann Machines," 27th International Conference on Machine Learning (ICML-10), 2010, pp. 807-814.

[31] O. Russakovsky, J. Deng, H. Su, J. Krause, S. Satheesh, S. Ma, Z. Huang, A. Karpathy, A. Khosla, M. Bernstein, A.C. Berg, and L. Fei-Fei, "ImageNet Large Scale Visual Recognition Challenge," Int. J. Comput. Vis., Vol. 115, pp. 211-252, 2015.

[32] T.-Y. Lin, M. Maire,S. Belongie, J. Hays, P. Perona, D. Ramanan, P. Dollár, and C.L. Zitnick, "Microsoft COCO: Common Objects in Context," European Conference on Computer Vision, 2014, pp. 740-755.

[33] F. Chollet, "Xception: Deep Learning with Depthwise Separable Convolutions," IEEE Conference on Computer Vision and Pattern Recognition (CVPR), 2017, pp. 1800-1807.

[34] J. Deng, W. Dong, R. Socher, L. Li, K. Li, and L. Fei-fei, "ImageNet: A Large-Scale Hierarchical Image Database," 2009 IEEE Conference on Computer Vision and Pattern Recognition, 2009, pp. 248-255.

[35] C. Szegedy, S. Ioffe, V. Vanhoucke, and A. Alemi, "Inception-v4, Inception-ResNet and the Impact of Residual Connections on Learning,'
Thirty-First AAAI Conference on Artificial Intelligence (AAAI'17), 2017, pp. 4278-4284.

[36] B. Zoph, G. Brain, and J. Shlens, "Learning Transferable Architectures for Scalable Image Recognition," IEEE/CVF Conference on Computer Vision and Pattern Recognition, 2018, pp. 8697-8710.

[37] K. Simonyan and A. Zisserman, "Very Deep Convolutional Networks for Large-Scale Image Recognition," International Conference on Learning Representations, 2015, pp. 1-14.

[38] K. Radhika, K. Devika, T. Aswathi, P. Sreevidya, V. Sowmya, and K.P. Soman, "Performance Analysis of NASNet on Unconstrained Ear Recognition," in Nature Inspired Computing for Data Science, M. Rout, J. K. Rout, and H. Das, Eds., New York, USA: Springer International Publishing, 2020, pp. 57-82.

[39] G. Huang, Z. Liu, L. Van Der Maaten, and K.Q. Weinberger, "Densely Connected Convolutional Networks," IEEE Conference on Computer Vision and Pattern Recognition (CVPR), 2017, pp. 2261-2269.

[40] T. Kohonen, "Self-organized Formation of Topologically Correct Feature Maps," Adv. Comput. Neurosci. Control Inf. Theory Biol. Syst., Vol. 43 No. 1, pp. 59-69, 1982.

[41] J. Yosinski, J. Clune, Y. Bengio, and H. Lipson, "How Transferable are Features in Deep Neural Networks?" Proc. 27th International Conference on Neural Information Processing Systems, 2014, Vol. 2, pp. 3320-3328.

[42] A. Sharif, R. Hossein, A. Josephine, and S. Stefan, "CNN Features Offthe-Shelf: An Astounding Baseline for Recognition," IEEE Conference on Computer Vision and Pattern Recognition Workshops, 2014, pp. 512 519.

[43] B. Zhou, A. Lapedriza, J. Xiao, A. Torralba, and A. Oliva, "Learning Deep Features for Scene Recognition using Places Database," Advances in Neural Information Processing Systems 27 (NIPS 2014), 2014, pp. 487-495. 